\title{
Updating and feature overwriting in short-term memory for timbre
}

\author{
Tom Mercer And Denis MCKeown \\ University of Leeds, Leeds, England
}

\begin{abstract}
Previous research has demonstrated a potent, stimulus-specific form of interference in short-term auditory memory. This effect has been interpreted in terms of interitem confusion and grouping, but the present experiments suggested that interference might be a feature-specific phenomenon. Participants compared standard and comparison tones over a 10 -sec interval and were required to determine whether they differed in timbre. A single interfering distractor tone was presented either $50 \mathrm{msec}$ or $8 \mathrm{sec}$ after the offset of the standard (Experiment 1) or $2 \mathrm{sec}$ prior to its onset (Experiment 2). The distractor varied in the number of features it shared with the standard and comparison, and this proved critical, since performance on the task was greatly impaired when the distractor either consisted of novel, unshared features (Experiment 1) or contained the distinguishing feature of the comparison tone (Experiments 1 and 2). These findings were incompatible with earlier accounts of forgetting but were fully explicable by the recent timbre memory model, which associates interference in short-term auditory memory with an "updating" process and feature overwriting. These results suggest similarities with the mechanisms that underlie forgetting in verbal short-term memory.
\end{abstract}

Short-term memory, the "active, but analyzed, contents of mind" (Nairne, 2003, p. 423), refers to the internal record of immediate experience and is a crucial component of human cognitive processing (Jonides et al., 2008). Although much recent effort has been directed at understanding verbal short-term memory (e.g., Berman, Jonides, \& Lewis, 2009; Camos, Lagner, \& Barrouillet, 2009; Lewandowsky, Oberauer, \& Brown, 2009; Oberauer \& Lange, 2008), impressive advances have also been made in the study of nonverbal stores, such as visual short-term memory (e.g., Alvarez \& Cavanagh, 2008; Fougnie \& Marois, 2009; Zhang \& Luck, 2009). Progress in the auditory memory field, however, has been less significant. Indeed, a recent review of the literature concluded that many fundamental questions pertaining to this store remain unanswered (Demany \& Semal, 2008), and this is particularly apparent in research into the mechanisms underlying forgetting in short-term auditory memory. Although numerous studies have suggested that an auditory memory trace simply decays over the passage of time, this explanation is now encountering increasing doubt. Initial support for the notion of time-based forgetting was derived from experiments using the two-tone comparison paradigm - a procedure requiring participants to discriminate two tonal stimuli over a silent retention interval of variable duration. Multiple studies employing this methodology have documented a clear decline in task performance as the retention interval separating the tones increased, seemingly reflecting temporal decay (e.g., Bachem, 1954; Clément, Demany, \& Semal, 1999; Harris, 1952; Kaernbach \& Schlemmer, 2008).
However, Cowan, Saults, and Nugent $(1997,2001)$ have offered an alternative explanation of this time-based decline. They noted that the majority of studies using the two-tone comparison procedure had extended retention intervals separating tones (up to $20-30 \mathrm{sec}$ ) but very brief gaps between trials (typically around 2-6 sec). This methodological flaw becomes apparent when trials with lengthy retention intervals are considered, because, in this situation, the first tone within a pair is temporally closer to the second tone of the previous trial, rather than TO the tone it actually has to be compared with. Inadvertent proactive interference from past trials may therefore have induced the performance decrement observed over long intervals, and support for this notion was provided by Cowan et al. (2001) in a reexamination of their 1997 data. They found no evidence of any information loss when tones on the current trial were maximally separated from preceding trials - regardless of the retention interval duration. Although this could be attributed to temporal distinctiveness - preserving a role for time in forgetting (see Lewandowsky, Brown, Wright, \& Nimmo, 2006, for an overview) - additional studies have more directly implicated interference from past events in the loss of shortterm auditory memory.

For instance, Ruusuvirta, Wikgren, and Astikainen (2008) used a simple two-tone comparison task and required their participants to judge whether the second tone within a trial (the "comparison") was higher or lower in pitch than the first tone (the "standard"). This judgment was heavily influenced by past events, because 
performance was significantly impaired when the pitch difference between the standard and the comparison of the previous trial was in the same direction as that of the current trial. A reverse facilitatory effect was revealed when the pitch difference between the two tones was in the opposite direction (see also Ruusuvirta, 2000; Ruusuvirta, Astikainen, \& Wikgren, 2002). Further evidence for proactive interference was provided by Visscher, Kahana, and Sekuler (2009), who presented individuals with two broadband sounds followed by a probe. The participants had to decide whether the probe matched either of the broadband sounds, and, in an impressive demonstration of the carryover of item information, Visscher et al. reported significantly higher false alarm rates when the broadband stimuli from the previous trial were similar to the probe on the current trial.

Disruption to short-term auditory memory can also be produced by subsequently occurring events (i.e., retroactive interference), as is evidenced by the interpolated-tone paradigm (Deutsch, 1970). In this procedure, listeners compare standard and comparison tones over an interval containing a number of interfering distractor tones. Although participants need not attend to the distractors, it is well documented that they can severely degrade performance when they are perceptually similar to the standard (see Deutsch, 1999, for a review). Such stimulus-specific retroactive interference has been examined largely within the context of pitch memory, but Starr and Pitt (1997) have revealed an analogous effect in memory for timbre, or "sound quality." Their participants compared the two target tones over a 5 -sec interval, while ignoring a sequence of six identical interpolated distractor tones. A striking stimulus-specific interference effect was uncovered, with accuracy on the task decreasing by $13 \%$ when the standard and distractors were perceptually similar in their harmonic (spectral) composition. Performance gradually improved as the distractors became more distinct from the standard, eventually matching the accuracy shown in the baseline (no interference) condition. This impressive finding seemingly confirms that the magnitude of retroactive interference is determined by the perceptual similarity of the interfering distractor tones and the standard. Such an effect appears to play a salient role in proactive interference too (see Visscher et al., 2009).

Intuitively, it would appear that perceptually similar distractors can blur the memory of the standard, thereby distorting the accuracy of the trace (Pechmann \& Mohr, 1992; Semal, Demany, Ueda, \& Hallé, 1996), and Ruusuvirta et al. (2002) have argued that the interfering tones bias the representation of the existing memory toward the frequency of the interfering event. Building on these ideas, Ruusuvirta et al. (2008) asserted that interference is a consequence of interitem confusion. That is, the presence of interfering distractors introduces difficulty in remembering when the standard occurred in relation to other tones (see also Deutsch, 1984). Support for this hypothesis was derived from studies that incorporated either the standard or comparison into the distractor tone sequence itself. Presenting the standard as an interfering tone typically facilitates performance, but both Deutsch (1972a) and Ries and
DiGiovanni (2009) established that accuracy on the task was poorest when the comparison tone also occurred as a distractor. According to interitem confusion, presentation of the comparison within the distractor sequence introduces temporal order confusion, leading participants to conclude erroneously that the distractor comparison was actually the standard (Deutsch, 1984).

Although plausible, alternative explanations of similarity-based interference emphasize the role of grouping and streaming mechanisms. Ueda (2004) noted that perceptual similarity, ostensibly so critical to interference, is actually very closely related to perceptual organization, and Jones, Macken, and Harries (1997) have suggested that "interference" in auditory memory may essentially reflect the degree to which the standard and distractors are perceptually integrated or grouped. The likelihood of grouping is heavily dependent on the auditory context in which sounds occur, particularly the temporal context (see Snyder, Carter, Lee, Hannon, \& Alain, 2008; Winkler et al., 2003), and Jones et al. recognized that the temporal arrangement of the typical interpolated-tone paradigm task may have established a context extremely favorable for the operation of grouping since many studies employing this procedure used an interval between the standard and first distractor, which was identical to that separating the distractor tones themselves (often $300 \mathrm{msec}$; e.g., Deutsch, 1970; Starr \& Pitt, 1997). This might have led to the standard and the distractors being organized into one perceptual unit. The decline in performance in many earlier experiments may therefore have been a consequence of the difficulty disentangling and isolating the standard from the interfering tones, rather than genuine memory trace interference. To test this notion, in one experiment, Jones et al. presented nine distractor tones during the retention interval. Each distractor was separated by $350 \mathrm{msec}$ and, crucially, the gap separating the standard from the first distractor was also varied. This latter interval was $1,500 \mathrm{msec}$ in one condition, ensuring that the standard and distractors were temporally distinct and grouping was minimized. But in the second condition, this interval was $350 \mathrm{msec}$ - that is, a duration equal to the gap dividing the distractors. This increased the likelihood of the standard merging with the distractor sequence, and Jones et al. did find that discrimination accuracy was significantly lower in the 350 -msec condition than in the 1,500-msec condition.

This grouping explanation indicates that both the spectral similarity and the temporal proximity of the standard and distractors determine the magnitude of disruption, but some studies have revealed interference where the possibility of grouping is unlikely. For example, Deutsch (1978a) required participants to compare standard and comparison tones over an interval containing six distractors. These distractors were chosen either from within the same octave as the two target sounds or from a wider 2 -octave range. A grouping account would anticipate greater interference when the distractors and standard were proximal in frequency (i.e., when they were derived from the same octave), but Deutsch actually uncovered a larger number of errors when the distractors came from the 2-octave range. Additionally, Ries and DiGiovanni (2007) 
found strong interference when the standard and the first distractor were separated by an interval of over $1 \mathrm{sec}$ (i.e., temporally distant stimuli). Such findings do not indicate that there is no role for perceptual organization in auditory memory, but they do demonstrate that grouping alone is incapable of accounting for all demonstrations of interference in short-term auditory memory. Other mechanisms must surely play a role. In this regard, the interference process posited by McKeown and Wellsted's (2009) recent timbre memory model (TMM) seems promising. TMM is inspired by the vast literature on mismatch negativity (MMN), an event-related potential elicited in response to small changes within a regular auditory sequence. For example, in the typical "oddball" experiment, the MMN is elicited when a series of standard tones is interrupted by an unexpected deviant (Winkler \& Cowan, 2005). One potential explanation suggests that the auditory system establishes and maintains a model of the recent acoustic environment in order to generate predictions about future events (Winkler, 2007; Winkler, Karmos, \& Näätänen, 1996). Any detected irregularities indicated by MMN require the model to be adjusted through an "on-line" updating process, which ensures that an up-to-date representation of the auditory environment is maintained (Horváth et al., 2008; Sussman \& Winkler, 2001; Winkler et al., 1996). This model-adjustment hypothesis is the most common interpretation of MMN (Garrido, Kilner, Stephan, \& Friston, 2009) and has been used to inform certain aspects of the TMM framework. According to TMM, recent acoustical information is stored within a contextualized auditory memory in the form of a spectral code. This code comprises different features - that is, the frequency and amplitude descriptions of the components of a complex sound. Features that are temporally synchronous cohere to define different auditory objects, and, by maintaining these objects over tens of seconds, TMM posits that a model of the recent auditory environment is established (McKeown \& Wellsted, 2009; Mercer \& McKeown, 2010). Of course, this model must be updated when novel events occur, in order to keep an accurate record of the latest changes within the auditory context. Forgetting is therefore a consequence of the model being updated by novel events (Mercer \& McKeown, 2010).

This updating process is of central importance to TMM, since it allows newly encountered stimuli to be entered into the memory model. TMM further suggests that updating is mediated via stimulus-specific adaptation. There is already some evidence supporting the relationship between cortical adaptation and auditory memory, with Ulanovsky, Las, Farkas, and Nelken (2004) demonstrating stimulusspecific adaptation in cat auditory cortex that could endure for tens of seconds. They suggested that this form of adaptation is a correlate of auditory sensory memory, which might also be involved in novelty detection (see Ulanovsky, Las, \& Nelken, 2003). Although the animals in Ulanovsky and colleagues' experiments were anesthetized, stimulus-specific adaptation has been shown in the auditory cortex of awake rats (von der Behrens, Bäuerle, Kössl, \& Gaese, 2009), and numerous other studies have documented persisting effects of prior stimulation, many of which appear to be manifest as adapted responses that are specific to the frequency of the prior tone (see McKeown $\&$ Wellsted, 2009, for a discussion of such aftereffects), although enhanced responses are sometimes observed too (e.g., Brosch, Schulz, \& Scheich, 1999). Additionally, the N1 response, which has been linked to adaptation (see Tarkka et al., 2002), is related to auditory memory (e.g., Lu, Williamson, \& Kaufman, 1992), and numerical simulations conducted by May and Tiitinen (2007) have shown that stimulus-specific adaptation may provide a memory mechanism that bestows sequence sensitivity to auditory cortex (see also May \& Tiitinen, 2010). Inspired by these ideas, TMM hypothesizes that previously experienced spectral features elicit an adapted response that is contrasted with the nonadapted response to novel features. This contrast permits the detection of changes within the auditory environment, since there will be a larger response to novel sounds. This notion fits neatly with the arguments of Jääskeläinen et al. (2004), who postulated that adapted responses to "old" sounds permit rapid and strong responses to novel stimuli. This allows new sounds to be quickly gated into conscious awareness where they can be acted on. However, the majority of experiments within the MMN literature have advocated a separate memorybased account, which is held to be distinct from explanations based on adaptation (e.g., Näätänen, Jacobsen, \& Winkler, 2005). Thus, the model-adjustment hypothesis (e.g., Winkler et al., 1996) and the adaptation hypothesis (e.g., Jääskeläinen et al., 2004) typically have been treated as independent explanations of $\mathrm{MMN}$, and an ongoing debate is questioning whether MMN is better interpreted as being a latency- and amplitude-modulated version of the N1 response (in line with the notion of adaptation) or is actually a separate component (see May \& Tiitinen, 2010). Our own conception is that the internal memory model of the recent acoustic environment is mediated by adaptation. TMM is, therefore, more congruent with May and Tiitinen's (2010) postulation that change detection in auditory memory is bestowed by stimulus-specific adaptation and that auditory memory may be manifested physiologically as adaptation in cortical cells.

The importance of adaptation becomes clearer when one considers behavioral tasks that require listeners to distinguish among standard and comparison tones over an interval containing one or more interfering distractors. Distractors comprising previously encountered features (i.e., repeated spectral components) do not require the model to be updated and instead simply produce an adapted response. These distractors are expected to have very little impact on behavioral performance. Conversely, novel-feature distractors $d o$ require the model to be updated, producing a nonadapted response and reducing task performance. It may be assumed that participants actively try to maintain an image of the standard tone during memory tasks - most probably by focusing attention on the representation (i.e., a nonverbal, attentional rehearsal process; see Camos et al., 2009; Cowan \& AuBuchon, 2008; Hourihan, Ozubko, \& MacLeod, 2009). However, as the model is updated in response to the occurrence of novel features, greater weight is given to these newly ex- 
perienced sounds, and this reduces the salience of objects already held in memory (i.e., the maintenance of the existing memory trace is disrupted). This degrades accuracy on the task. Nonetheless, the impact of novel features is assumed to differ depending on how similar the interfering features are to those already held within memory. That is, whereas any novel feature should cause impairment, maximal interference is produced by distractors containing novel features that are very close to those currently held in memory. Such distractors elicit the updating process and also selectively overwrite certain features currently held in memory (Mercer \& McKeown, 2010). This distorts the existing trace and increases the difficulty of the discrimination. TMM therefore posits that an updating process, with the possibility of additional feature overwriting, underlies interference in short-term auditory memory.

Although there is evidence for the role of feature-based interaction in pitch memory (e.g., Deutsch, 1978b, 1982; Deutsch \& Roll, 1974), support for the TMM account was recently provided by Mercer and McKeown (2010). Their participants were required to compare standard tones with comparison tones, varying in timbre, over a 4.7-sec interval. Similar to Starr and Pitt (1997), Mercer and McKeown manipulated the spectral profile of all the timbres within the experiment, varying the harmonic (feature) composition of 15 complex tones. Each tone contained the fundamental and 8th harmonic, but two other harmonics were free to vary. The standard and comparison tones differed by a single feature (the 6th or 7th harmonic). For example, the 6th harmonic presented in the standard tone might shift to the 7th harmonic in the comparison on different trials. These were termed critical features, since they were crucial to performing the discrimination. A single distractor, varying in the number of features it shared with the two target tones, was presented 0,100 , or 1,200 msec after the offset of the standard. In line with TMM, distractors that contained the critical, repeated feature of the standard tone (the 6th harmonic, in the example above) did not significantly reduce performance in comparison with no interference. This is compatible with the notion of an adapted response. Conversely, distractors that contained novel features (i.e., features that were not incorporated into either the standard or comparison) did impair discriminatory accuracy. The greatest disruption occurred when the distractor contained the critical distinguishing feature of the comparison tone (the 7th harmonic, in the example above). This was interpreted as a feature-specific overwriting process, whereby the critical comparison feature presented in the distractor selectively overwrote the critical standard feature. This interference effect occurred regardless of the temporal position of the distractor, and Mercer and McKeown concluded that, rather than perceptual similarity per se, it was the presence of certain features within the distractor that caused the drop in participants' discriminatory ability. Still, a more convincing test of this notion would present individual features, rather than multicomponent complex tones, as distractors. Mercer and McKeown (and almost all other experiments interrogating interference in auditory memory) used standards and distractors with an identical number of components. This arrangement makes it problematic to dissociate general (broadly defined) similarity-based confusion from featurespecific (narrowly defined) interference, and additional issues, such as the role of consolidation and the impact of proactive interference, still warrant further attention. Thus, we designed the present experiments to address these issues and to provide more thorough tests of TMM and of the nature of interference in auditory memory.

\section{EXPERIMENT 1}

We designed Experiment 1 to examine whether retroactive interference in timbre memory was a feature-specific, time-invariant phenomenon. Participants compared standard and comparison tones over a 10-sec interval and decided whether they were the same or different. As in our previous study (Mercer \& McKeown, 2010), tones varied in spectral timbre, but the method of manipulating this acoustic property differed. Although in earlier experiments the position of the harmonics was varied (e.g., Mercer \& McKeown, 2010; Starr \& Pitt, 1997), in the present experiment, the intensity of a single component was elevated within each complex tone. This raised component defined the timbre of the complex and acted as the critical feature (i.e., the component crucial to successful discrimination). On different trials, the critical feature of the comparison was always immediately adjacent to the critical standard feature. For example, one standard had the fourth component incremented, whereas its associated comparison on a different trial had the fifth component elevated. A single distractor tone, comprising just two equal-amplitude harmonics, occurred during the retention interval and varied in the extent to which it overlapped with the features of the standard and the comparison. Since the two target tones comprised six harmonics, the presence of distractors with just two components - very different from the multicomponent tones being compared - allowed a more thorough assessment of TMM's notion that specific features are responsible for interference.

Five types of distractor stimulus were created and are shown in Figure 1 in an example trial. The first distractor (noncritical overlap) was within the bandwidth of the two target tones, but did not overlap with either of the critical features. Figure 1 shows the critical feature of the standard as the 4th harmonic and the critical feature of the comparison as the 5th. In this scenario, the noncritical-overlap distractor therefore contained the 1st and 2nd harmonics. The standard-feature distractor comprised the distinguishing feature of the standard tone and an adjacent noncritical harmonic (the 3rd and 4th harmonics). The reverse condition (the comparison feature distractor) incorporated the distinguishing feature of the comparison and an adjacent noncritical harmonic (the 5th and 6th harmonics). There was also a condition in which the distractor included the critical features of both the standard and the comparison (the 4th and 5th harmonics). The final distractor type, novel features, was outside of the bandwidth of the target complexes and contained the 8th and 9th harmonics.

The distractor could occur either $50 \mathrm{msec}$ or $8 \mathrm{sec}$ after the offset of the comparison - an arrangement that served 
Standard

123456
Distractor

$\begin{array}{lllllllll}1 & 2 & 3 & 4 & 5 & 6 & 7 & 8 & 9\end{array}$
Comparison

123456

Distractor Type

1: Noncritical overlap
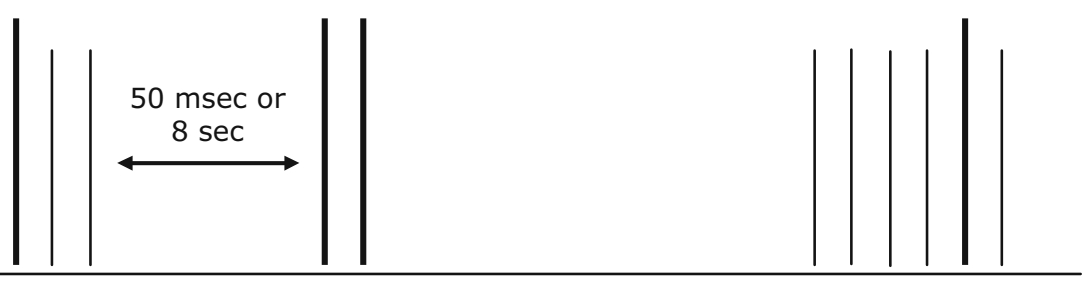

$\begin{array}{lllllllll}1 & 2 & 3 & 4 & 5 & 6 & 7 & 8 & 9\end{array}$

2: Standard feature

$\begin{array}{lllllllll}1 & 2 & 3 & 4 & 5 & 6 & 7 & 8 & 9\end{array}$

3: Comparison feature

4: Both critical features

$\begin{array}{lllllllll}1 & 2 & 3 & 4 & 5 & 6 & 7 & 8 & 9\end{array}$

$\begin{array}{lllllllll}1 & 2 & 3 & 4 & 5 & 6 & 7 & 8 & 9\end{array}$

\section{5: Novel features}

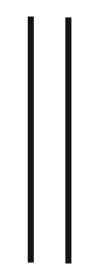

Figure 1. Diagrammatic representation of the distractor types used in Experiment 1. Participants compared standard and comparison complex tones over a 10-sec interval and decided whether they were the same or different. A single distractor tone, comprising two harmonics, occurred either 50 msec or $8 \mathrm{sec}$ after the offset of the standard and varied in the degree to which it overlapped with the two target sounds. See text for details.

to address whether interference could be alleviated following consolidation (see Wixted, 2004). Consolidation theory suggests that newly created memories initially remain transient and vulnerable until they are stabilized over time (McGaugh, 2000), and Massaro (1970) was among the first to demonstrate this possibility in auditory memory. In the usual procedure, his listeners compared standard and comparison tones over a retention interval and made a same/different judgment. During this interval, a distractor tone lasting $0.5,1,2$, or 4 sec occurred, either immediately after the offset of the standard (preventing consolidation) or after an 800-msec gap (permitting consolidation). Performance was greatly improved when a period of silence preceded the onset of the interferer, suggesting that the memory trace became more resistant to interference after it had been consolidated. A more recent study by Berti, Münzer, Schröger, and Pechmann (2006) also implicated a role for consolidation. They varied the interval separating the standard and the first distractor between 150 and $1,450 \mathrm{msec}$ in the interpolated-tone paradigm and found significantly better performance with longer gaps (see also Elliott, 1970, for similar findings). However, Mer- 
cer and McKeown (2010) reported time-invariant interference: Performance was equally disrupted, regardless of whether the distractor occurred 0,100 , or $1,200 \mathrm{msec}$ after the standard. Still, it is possible that $1,200 \mathrm{msec}$ does not offer enough time for the complete consolidation of complex timbral stimuli; therefore, we employed an extended gap $(8 \mathrm{sec})$ between the standard and distractor in the present study. Research suggests that a memory trace is encoded within $250 \mathrm{msec}$ from stimulus onset (e.g., Massaro, 1972), and all tones were $200 \mathrm{msec}$ in duration in the present study. Thus, although the auditory memory trace was properly encoded before a distractor occurred at the 50-msec interval, there was little time for consolidation. However, in the 8-sec condition, there was ample time for the trace to be strengthened and protected from interference. A consolidation account would therefore anticipate alleviated interference after $8 \mathrm{sec}$. In contrast, TMM predicted equivalent interference between the 50msec and 8-sec conditions, and this time-invariant interference was hypothesized to be a consequence of certain types of features presented in the distractor. Distractors in the noncritical-overlap and standard-feature groups were predicted to have limited impact on performance, since they are simply repeating features already maintained within the memory buffer, producing an adapted response. Conversely, distractors containing the critical comparison feature or novel features were expected to severely impair accuracy on the task in relation to the no interference control. This is exactly what happened.

\section{Method}

Participants. Four individuals ( 2 female, mean age $=23.75$, range 21-27 years), with self-reported normal hearing and prior experience of psychoacoustical procedures, took part in the study. One was the first author. The remaining 3 participants received hourly payment for participation. Only 1 participant was an experienced musician (U.K. Grade 8, with 13 years of formal training); the other 3 participants (including the first author) had little or no musical expertise and did not play any instrument.

Apparatus and Stimuli. Four complex tones comprising six harmonics were generated and served as the target tones. The individual frequency components were approximately $78 \mathrm{~dB}$ SPL in intensity, but a single harmonic within each complex was increased by a further $9.6 \mathrm{~dB}$ (see Figure 2). This raised component defined the timbre of each tone and was termed the critical feature. Five different types of distractor tone were also generated (noncritical overlap, standard feature, comparison feature, both critical features, and novel features). These consisted of just two equal-amplitude harmonics presented at the same intensity as the raised component of the target sounds. All stimuli were $200 \mathrm{msec}$ in duration, including a $10-\mathrm{msec}$ cosine onset and offset ramp, and could be presented at any of nine pitches $(\mathrm{C}$ at $130.8 \mathrm{~Hz}$, through $\mathrm{C} \#, \mathrm{D}, \mathrm{E}, \mathrm{F}, \mathrm{F}, \mathrm{G}, \mathrm{A}$, and $\mathrm{A}$ at $220.0 \mathrm{~Hz}$ ). Pitch was held constant within a trial, and the tones were also roved in intensity both between $(0-9 \mathrm{~dB})$ and within $(0-6 \mathrm{~dB})$ trials.

Stimuli were created via TDT RP2.1 hardware and Mathwork's MATLAB software. We used a custom-designed program developed in MATLAB to present the trials and record participant responses. Tones were attenuated (TDT PA5), bandpass filtered (Kemo VBF21M filter: $100 \mathrm{~Hz}$ to $10 \mathrm{kHz}$ ), and output via a TDT HB7 headphone amplifier to the left earpiece of a set of STAX SR-303 Classic headphones. All participants completed the task within an Industrial Acoustics Company double-walled sound-attenuating booth. The component levels for all stimuli used within this experiment were

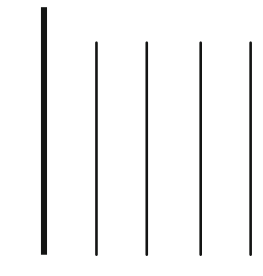

Timbre A

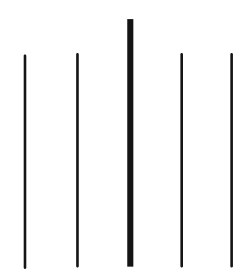

Timbre C

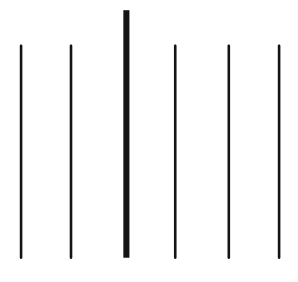

Timbre B

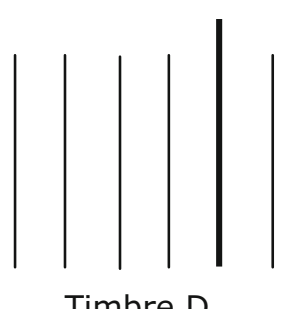

Timbre D
Figure 2. Diagrammatic representation of the target tones. Each tone comprised five equal-amplitude harmonics plus another component incremented by $9.6 \mathrm{~dB}$ SPL (taller bold line in figure). This incremented component changed the timbre of each complex and was termed the critical feature. Each of the four tones served as both the standard and comparison.

measured using a Brüel \& Kjær $2260 \mathrm{H}$ spectrum analyzer, running BZ7208 software, and a model 4153 artificial ear.

Design. Listeners had to compare standard and comparison tones, separated by an interval of $10 \mathrm{sec}$, and decide whether they were the same or different. As is shown in Figure 2, each of the four target tones could serve as both the standard and the comparison, but, to ensure that the difference between sounds was equivalent irrespective of trial type (i.e., that the target component was shifted by only one harmonic on different trials), Timbre A was paired with Timbre B and Timbre $C$ was paired with Timbre $D$. This pairing yielded eight trial types: $\mathrm{AA}, \mathrm{BB}, \mathrm{CC}, \mathrm{DD}$ (same trials) and $\mathrm{AB}, \mathrm{BA}, \mathrm{CD}$, and $\mathrm{DC}$ (different trials). Participants indicated their "same" or "different" judgment using a response box, and the next trial began $22 \mathrm{sec}$ after a buttonpress. This extended intertrial interval was intended to minimize the influence of previous trials (see Cowan et al., 1997).

Given the nature of the stimuli, one of our concerns was that listeners might rely on an overall difference in loudness between the standard and the comparison to perform the discrimination. To combat this, we introduced within-trial roving of intensity to remove any potential loudness cues and to ensure that the listener was discriminating the sounds on the basis of spectral shape (Green, Kidd, \& Picardi, 1983; Kidd \& Mason, 1992). That is, standard and comparison tones were presented at different intensities within a trial, and participants were instructed to ignore loudness and to differentiate the tones solely on the basis of timbre. Intensity and pitch were also varied between trials to minimize the possibility that listeners were relying on a form of context coding (Durlach \& Braida, 1969) or developing long-term memories of the stimuli. Instead, participants had to rely on a nonverbal representation of the standard. There was only a very small difference between the target tones, and all the timbres sounded "abstract." This guaranteed that listeners were not verbally encoding or labeling the sounds, and no participant reported using such a strategy. Roving pitch and loudness on a trial-by-trial basis also prevented listeners from simply segregating the critical component from the standard and comparison complexes and contrasting these harmonics directly (rather than listening synthetically to the "global" timbre of the tone).

Procedure. In the first phase of the experiment, participants were required simply to compare tones over a silent interval. The intention was to ensure that listeners were able to reliably discriminate 
the tones; consequently, each participant had to attain a minimum of $75 \%$ correct across two stimulus blocks before proceeding to the second phase. Reflecting the difficult nature of the discrimination, an average of $5.5 \mathrm{~h}$ was required before listeners attained criterion performance (range: $3.5-9.5 \mathrm{~h}$ ). In the second phase, we introduced a single distractor tone, which varied in its overlap with the features of the standard and the comparison. This interfering tone occurred either $50 \mathrm{msec}$ or $8 \mathrm{sec}$ after the offset of the standard, but in the no-interference control, the distractor was removed and listeners compared target tones over a silent interval.

The second phase of testing lasted over $8 \mathrm{~h}$, but was completed in individual 30-min sessions over a period of several weeks. Each session comprised two stimulus blocks, and a block contained 24 trials arranged in a random order (12 same, 12 different). Each major trial type (AA, BB, CC, DD, AB, BA, CD, and DC) was presented three times in a block, with each distractor type occurring at least four times. Within each session, listeners attempted both the $50-\mathrm{msec}$ and 8 -sec conditions in separate stimulus blocks, but in three cases, one of these conditions was replaced by the no-interference control. There were 40 individual trial types (eight trial types $\times$ five distractor types), each of which was presented nine times at nine different pitches. Participants completed a total of 792 experimental trials during this second phase, and no feedback was provided.

\section{Results}

We calculated $P_{\mathrm{SS}}$ (the proportion of "same" responses on same trials) and $P_{\mathrm{SD}}$ (the proportion of "same" responses on different trials) values for each participant in the six interference conditions. These scores were then averaged across each participant and were used to generate $d^{\prime}$, the bias-free index of sensitivity (see Bi, 2002, for details on computing $d^{\prime}$ for the same-different methodology). This yielded five $d^{\prime}$ values for each distractor type in the 50msec and 8-sec conditions, plus $d^{\prime}$ for the no-interference control. These values are shown in Figure 3. Table 1 shows individual participant data; Participant 1 was author T.M.
Figure 3 shows a clear decrease in performance for all distractor types in comparison with the no-interference control, but the magnitude of this decline differed, depending on the condition. Distractors containing both critical features, the comparison feature, or novel features led to the greatest drop in discrimination accuracy, especially when they were presented at $50 \mathrm{msec}$. To assess the reliability of these trends, we compared the $d^{\prime}$ values in each condition with the control, using a technique described by Gourevitch and Galanter (1967; see also Macmillan \& Creelman, 2005). Gourevitch and Galanter's statistic is calculated by dividing the difference between two $d^{\prime}$ scores by the square root of the summed variance for the $d^{\prime}$ values (i.e., a $Z$ test). The outcome is approximately normally distributed and can, therefore, be assessed for significance (e.g., McKeown \& Wellsted, 2009; Nosofsky, 1983; Oberle \& Amazeen, 2003).

Employing this analysis for the 50-msec condition revealed that the noncritical-overlap and the standard-feature distractors did not differ from the no-interference control (noncritical overlap, $Z=-1.55, p=.121$; standard feature: $Z=-1.42, p=.156$ ). However, performance with distractors containing both critical features, the comparison feature, and novel features was significantly lower than control (both critical features, $Z=-2.34, p=.019$; comparison feature, $Z=-2.62, p=.009$; novel features, $Z=-2.53, p=.011)$. An identical trend was uncovered in the 8-sec condition. Performance with the noncriticaloverlap and standard-feature distractors did not differ from control (noncritical overlap, $Z=-1.4, p=.162$; standard feature, $Z=-1.33, p=.184$ ), but $d^{\prime}$ in the other conditions was significantly worse than it was for no interference (both critical features, $Z=-2.10, p=.036$; com-

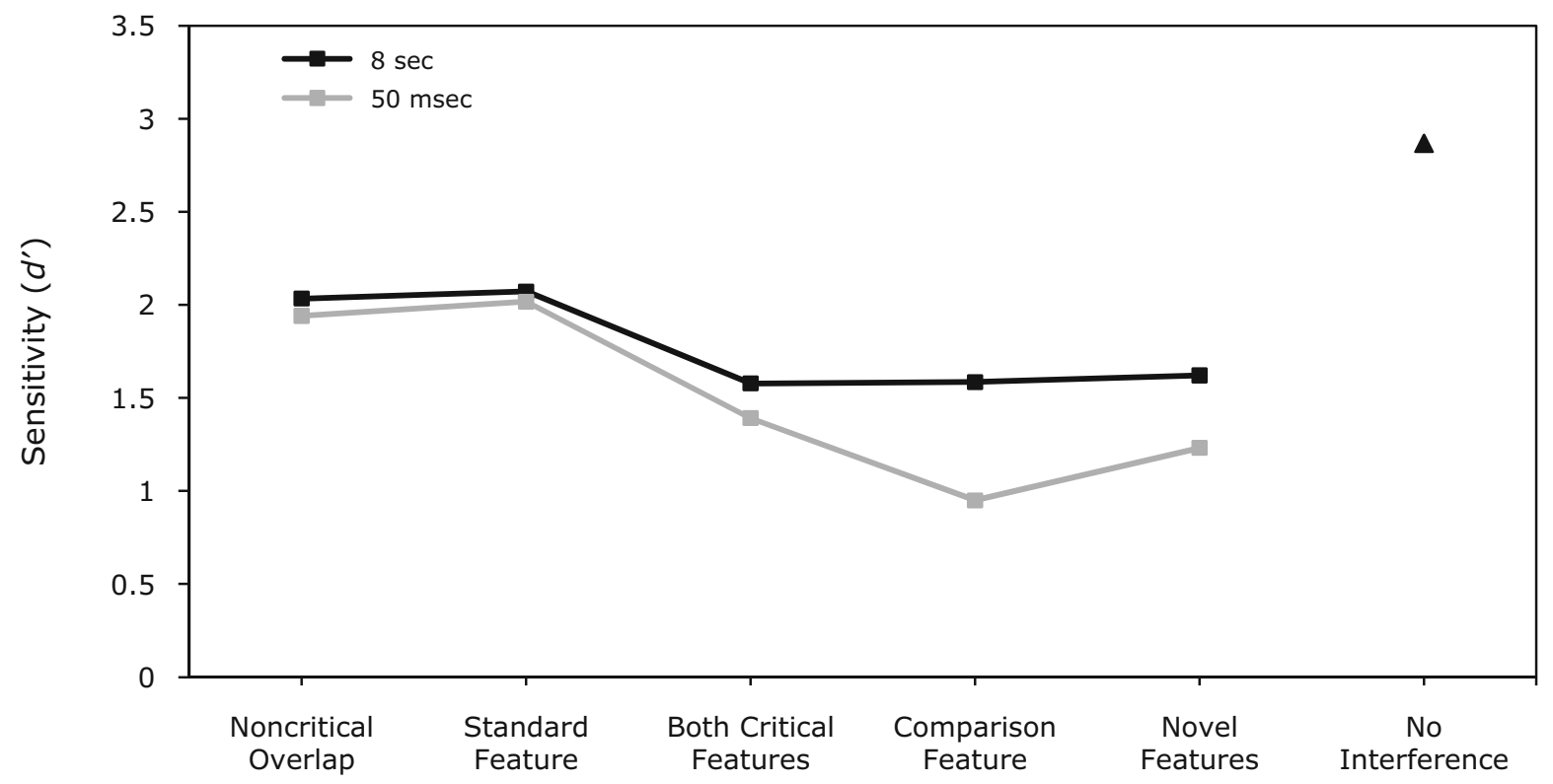

Distractor Type

Figure 3. Group $d^{\prime}$ values for the six conditions of Experiment 1. The gray line represents the 50-msec interval, and the black line represents the 8-sec interval. 
Table 1

Individual $d^{\prime}$ Values for Each of the Conditions in Experiment 1

\begin{tabular}{|c|c|c|c|c|c|c|c|c|}
\hline \multirow{2}{*}{$\begin{array}{l}\text { Interference } \\
\text { Condition }\end{array}$} & \multicolumn{2}{|c|}{ Participant 1} & \multicolumn{2}{|c|}{ Participant 2} & \multicolumn{2}{|c|}{ Participant 3} & \multicolumn{2}{|c|}{ Participant 4} \\
\hline & $50 \mathrm{msec}$ & $8 \mathrm{sec}$ & $50 \mathrm{msec}$ & $8 \mathrm{sec}$ & $50 \mathrm{msec}$ & $8 \mathrm{sec}$ & $50 \mathrm{msec}$ & $8 \mathrm{sec}$ \\
\hline Noncritical overlap & 1.501 & 1.027 & 1.630 & 1.343 & 3.195 & 3.210 & 2.290 & 3.578 \\
\hline Standard feature & 1.882 & 1.557 & 0.508 & 1.845 & 3.048 & 3.210 & 2.176 & 2.264 \\
\hline Both critical features & 1.920 & 1.447 & 0.848 & 1.713 & 2.047 & 2.051 & 1.007 & 1.546 \\
\hline Comparison feature & 0.539 & 1.183 & 1.349 & 1.296 & 1.572 & 2.555 & -0.484 & 1.713 \\
\hline Novel features & 0 & 1.422 & 0.304 & 0.666 & 2.047 & 2.594 & 1.263 & 1.906 \\
\hline No interference & \multicolumn{2}{|c|}{2.703} & \multicolumn{2}{|c|}{2.382} & \multicolumn{2}{|c|}{4.066} & \multicolumn{2}{|c|}{3.402} \\
\hline
\end{tabular}

parison feature, $Z=-2.10, p=.036$; novel features, $Z=$ $-2.03, p=.042)$. Comparison of the distractors across interval (i.e., $50 \mathrm{msec}$ vs. $8 \mathrm{sec}$ ) revealed no significant trends, and, even when performance was averaged across type of distractor, there was no reliable difference between results for conditions $50-\mathrm{msec}$ and 8 -sec conditions $(Z=$ $-0.43, p=.667)$.

To further explore these data, we conducted a $Q^{\prime}$ (see Michael, 2007). This statistic is an extension of an algorithm reported by Marascuilo (1970), and, in addition to permitting analysis of main effects, it allows interactions to be explored in $2 \mathrm{xK}$ designs. Confirming the previous analysis, the $Q^{\prime}$ test detected a significant main effect of distractor type $\left[Q^{\prime}(5)=18.01, p=.003\right]$. Multiple comparisons revealed that distractors containing repeated features (noncritical-overlap and standard feature) did not differ from control, but all three distractors comprising novel features (both critical features, comparison feature, novel features) significantly impaired performance in relation to no interference. This directly replicates the results of the $Z$ test. The $Q^{\prime}$ test also found a marginally significant effect of time $\left[Q^{\prime}(1)=3.65, p=.056\right]$, with performance at $8 \mathrm{sec}$ being slightly better than it was at $50 \mathrm{msec}$. There was no significant interaction. The main effect of time, which almost reached significance, was intriguing and appeared to contradict the earlier analysis. However, further exploration of the data suggested that this marginally significant effect was due largely to differences for the comparison-feature distractor, where performance did appear to recover slightly after $8 \mathrm{sec}$. Assessment of the individual data revealed that only 1 participant showed significantly worse performance with the comparison-feature distractor at $50 \mathrm{msec}$ compared with that at $8 \mathrm{sec}$ (see Table 1, Participant 4). Furthermore, a paired-samples $t$-test uncovered no significant difference for performance with this distractor type at the two intervals $[t(3)=2, p=.139]$, and Michael's (2007) multiple comparison procedure confirmed that performance at both $50 \mathrm{msec}$ and $8 \mathrm{sec}$ was significantly worse than it was for the silent (no interference) condition ( $p=.004$ and .035 , respectively).

\section{Discussion}

The results of the present experiment suggested that interference was a feature-specific phenomenon and that, overall, there was little role for consolidation. Slightly recovered performance after $8 \mathrm{sec}$ was largely confined to just a single condition (comparison feature), and only Par- ticipant 4 actually showed a reliable difference between the two intervals for this distractor. None of the group analyses reached significance, which contradicts earlier studies that found that discrimination accuracy reliably improved when the distractor tone sequence was temporally removed from the standard (e.g., Berti et al., 2006; Jones et al., 1997; Massaro, 1970). It is plausible that the improved performance after longer intervals documented by both Massaro (1970) and Berti et al. resulted partially from additional encoding time rather than from consolidation. Like the present study, Massaro (1970) employed stimuli that were $200 \mathrm{msec}$ in duration. His interference tone began either immediately or $800 \mathrm{msec}$ following the standard, and performance was better in this latter condition. Still, given that the time to encode acoustical stimuli is usually estimated as $250 \mathrm{msec}$ (Massaro, 1972), the interference tone in the immediate condition may have disrupted encoding processes. The beneficial effect Massaro (1970) found after $800 \mathrm{msec}$ of silence may therefore have been a consequence of the uninterrupted and complete encoding of the standard tone. Supporting this notion, Massaro (1970) did not find an advantage of a brief period of silence prior to the distractor when stimuli were $500 \mathrm{msec}$ in duration. Berti et al. used stimuli lasting for only $50 \mathrm{msec}$ and also found poorest performance when the distractor tones interrupted encoding. In the present study, a minimum of $250 \mathrm{msec}$ elapsed before the occurrence of the distractor; therefore, disruption to performance was unlikely to be due solely to interference with encoding. Perhaps there was partial disruption to encoding for some participants in the 50-msec condition (which would account for the marginally significant difference between the two intervals), but we believe that the bulk of the disruption was due to interference with the internal representation of the standard tone.

Other seminal differences also exist between the present study and the earlier experiments. For instance, Massaro (1970), Jones et al. (1997), and Berti et al. (2006) all required participants to make judgments on the basis of pitch, whereas the present experiment relied on listeners detecting a difference in spectral timbre. Both Jones et al. and Berti et al. also used multiple distractors, increasing the likelihood of establishing a grouping context. Last - and perhaps most important - the notion that there was no interference at the longer intervals in these earlier experiments is debatable. Jones et al. did not include a no-interference control, and, although Berti et al.'s design did incorporate this condition, it did not statistically as- 
sess how performance differed across interference and nointerference conditions. Despite this, Berti et al. did present performance in this control condition (their Figure 2, p. 114), and examination of their data clearly highlights a large drop in the proportion of correct responses at all intervals in comparison with no interference - approximately $30 \%$ for a subset of their sample at $1,450 \mathrm{msec}$. It therefore remains possible that there was still substantial interference at these longer intervals.

This was certainly the case in the present study, in which certain distractors greatly impaired performance, regardless of when they were presented. This time-invariant, retroactive interference was feature specific: Only distractors incorporating the critical feature of the comparison or novel features produced significant interference. Although this result challenges the pervasive assumption that interference is determined by perceptual similarity, the present findings do support the conjectures of TMM and the results of Mercer and McKeown (2010). In Experiment 2, we examined whether proactive interference was also feature specific.

\section{EXPERIMENT 2}

Although there have been a relatively large number of studies interrogating retroactive interference in short-term auditory memory (at least for pitch), the number of proactive interference studies is much smaller. Nonetheless, the recent acoustical past certainly can influence performance on current trials (e.g., Ruusuvirta, 2000; Ruusuvirta et al., 2002; Ruusuvirta et al., 2008; Visscher et al., 2009). This effect appears to be determined by the similarity between standard and distractors, and the most common explanation postulates that participants confuse the sequential order of the standard on the current trial and the comparison on the previous trial (interitem confusion; see Ruusuvirta et al., 2008). TMM also anticipates substantial impact of recent acoustical events, but suggests that this is a feature-specific process. Support for this idea can be found within the literature examining selective frequency listening and auditory attention. In a typical twoalternative forced choice task, participants are required to compare two complex tones and decide which one contained the target (usually an incremented component). It is now well established that a pure-tone cue occurring before the first tone can significantly affect performance (e.g., Dai, Scharf, \& Buus, 1991; Hafter, Schlauch, \& Tang, 1993; Hübner \& Hafter, 1995). For example, Green and McKeown (2001) discovered that cues that matched the frequency of the target substantially improved performance over trials in which the cue and target differed in frequency. This effect persisted, even when the interval between the cue and the first complex was $3 \mathrm{sec}$ and when the cue and target matched in frequency on only $12.5 \%$ of trials. In addition, Green and McKeown (2007, Experiment 2) revealed that target detection can be influenced by the cue from the previous trial. On trace trials, the target was at the same frequency as the cue from the previous trial; whereas, on no-trace trials, the target was presented at a frequency that had not occurred for at least four trials.
Impressively, mean performance on trace trials was about $6 \%$ higher than it was on no-trace trials.

In both of these experiments, the pretrial cue was a pure tone-a single feature - yet it was capable of affecting performance. Of course, these studies found a facilitatory impact of prior events, but strong evidence for the inhibitory nature of the pretrial cue does exist (see McKeown \& Wellsted, 2009). Building on this research, in Experiment 2, we aimed to examine whether proactive interference (e.g., the disruptive impact of previous tones) was feature specific. Participants compared standard and comparison tones over a silent 10 -sec interval while disregarding a distractor that occurred $2 \mathrm{sec}$ before the first tone. The distractor contained either the critical feature of the comparison or two novel features (8th and 9th harmonics). Both of these distractors had an adverse effect on discrimination accuracy in Experiment 1, but TMM generates different predictions for the proactive presentation of these tones. One of the central concepts underlying interference in TMM is the update process. In order to maintain an upto-date representation of the latest auditory environment, the adaptive model of TMM is necessarily maximally weighted by recent events, a process we term updating. But, even though newly occurring sounds are given "salience" by the model, the residue of old sounds can persist for tens of seconds. As such, old sounds can degrade performance in certain circumstances. In Experiment 1, the novel-feature distractor was capable of severely disrupting discriminatory ability, even though it did not overlap spectrally with the representation of the standard. That is, even though this distractor did not overwrite the record of the standard, it was weighted more highly than was prior auditory information that led to retroactive interference. It is the disruption of the salience of the prior record through updating that is responsible for interference from such novel features. But TMM assumes only distractors that overlap spectrally with the upcoming standard tone induce substantial proactive interference. That is, when the novel features distractor precedes the standard, it cannot produce updating, and, since it does not overlap with the spectral record of the succeeding standard, it cannot produce overwriting either. Hence, it should have negligible impact on performance, even though its residue persists during the retention interval. Conversely, the comparisonfeature distractor can cause interference when presented before the standard through a proactive feature overwriting mechanism. The residue of this distractor will persist and interact with the features of the standard, and, via proactive overwriting, the subsequent record of the standard will be impaired and discriminatory ability will be degraded. The results confirmed these predictions.

\section{Method}

Participants. The same individuals from Experiment 1 participated in the present study.

Design and Procedure. The standard and comparison were always separated by a silent 10 -sec interval, and the distractor tone now occurred $2 \mathrm{sec}$ prior to the standard. The intertrial interval was reduced to $20 \mathrm{sec}$. Only 2 distractor types were presented (the comparison feature and novel features), and this arrangement yielded 16 major trial types (8 standard-comparison pairings $\times 2$ distrac- 
tor types). However, in some cases, the distractor was removed in order to provide a measure of performance without interference (no-interference control). The entire experiment consisted of 216 individual trials divided into 24-trial stimulus blocks. Blocks containing a distractor included an equal number of novel features, and comparison feature tones were arranged in a random order. Blocks without a distractor (control) were undertaken separately. The study was completed in 30-min sessions over several weeks. All other aspects of the methodology were identical to Experiment 1 .

\section{Results}

As in Experiment $1, P_{\mathrm{SS}}$ and $P_{\mathrm{SD}}$ values were averaged across participants and were used to compute $d^{\prime}$ in the three conditions. The results, presented in Figure 4, demonstrate a striking drop in sensitivity for the comparisonfeature condition, but novel features appeared to have little impact. Indeed, task accuracy was reduced by an average of just $3.95 \%$ in the novel-features condition, but dropped $18.48 \%$ when the comparison-feature distractor occurred. Comparing the $d^{\prime}$ values with Gourevitch and Galanter's (1967) analysis revealed a nonsignificant difference between novel features and the no-interference control $(Z=-0.57, p=.569)$, but also revealed a highly significant decline in the comparison-feature condition $(Z=-2.27, p=.023)$. The results for individual participants are shown in Table 2 and highlight similar trends.

\section{Discussion}

Experiment 2 was designed to examine TMM's prediction that proactive interference in timbre memory is feature specific. The results appeared to confirm this hypothesis. Distractors containing the comparison feature had a very potent, harmful impact on performance, and although previous studies have already documented proactive interference, this has been limited typically to intervals of a few hundred milliseconds (e.g., Ruusuvirta et al., 2002; Ruusuvirta et al., 2008) or has been a relatively small effect (e.g., Visscher et al., 2009). Here, a strong proactive interference effect was uncovered for the comparison-feature distractor when it occurred $2 \mathrm{sec}$ before the standard. Novel features had barely any influence. These results are entirely in line with the TMM framework and will be discussed in greater depth below.

\section{GENERAL DISCUSSION}

In the present study, we examined whether interference in short-term auditory memory for spectral timbre was feature specific. Although prior explanations of interference have advocated interitem confusion (e.g., Deutsch, 1984; Ruusuvirta et al., 2008), grouping (e.g., Jones et al., 1997), or general trace distortion (e.g., Semal et al., 1996) as the underlying causal mechanisms, TMM hypothesizes that interference results from feature overwriting and updating (McKeown \& Wellsted, 2009; Mercer \& McKeown, 2010). To test this possibility, listeners compared standard and comparison tones over a 10 -sec interval. These target tones comprised six harmonics, one of which (i.e., the critical feature) was increased in intensity to manipulate the overall shape of the sound and thus alter its spectral timbre. A single distractor tone, varying in its spectral overlap with the standard and comparison, was presented either $50 \mathrm{msec}$ or $8 \mathrm{sec}$ after the standard (Experiment 1) or $2 \mathrm{sec}$ before its onset (Experiment 2). In the majority of previous experiments, the standard and distractors have consisted of an equal number of components (i.e., both were pure tones, or both were complex tones). This arrangement makes it extremely difficult to determine whether interference is a consequence of the overall perceptual similarity between the tones or due to a more specific, feature-interaction effect. Hence, in the present experiments, the distractor contained only two harmonics (features), permitting a more thorough examination of the potential feature-specific nature of interference.

In Experiment 1, distractors containing previously experienced, repeated spectral features (noncritical overlap and standard feature) did not significantly reduce per-

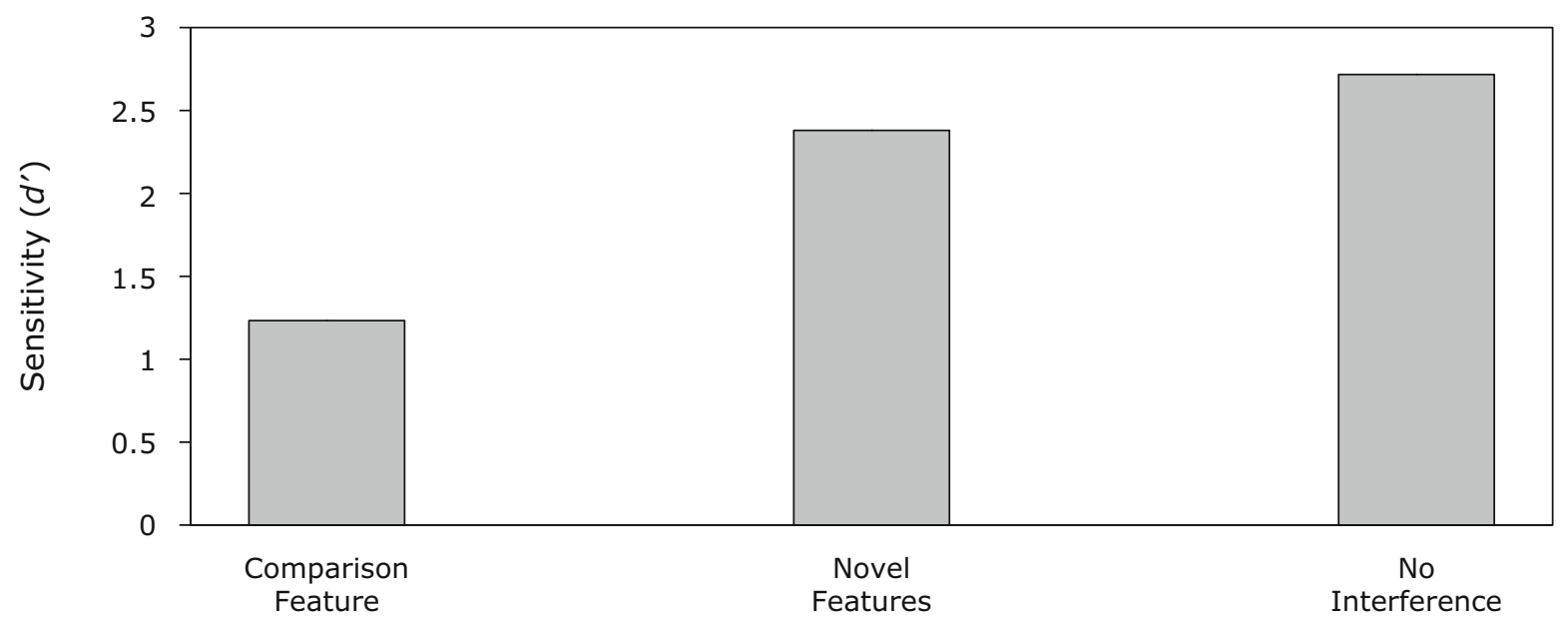

Distractor Type

Figure 4. Group $\boldsymbol{d}^{\prime}$ values for the three conditions of Experiment 2. 
Table 2

Individual $d^{\prime}$ Values for Each of the Conditions in Experiment 2

\begin{tabular}{lcccc}
\hline \multicolumn{1}{c}{ Condition } & Participant 1 & Participant 2 & Participant 3 & Participant 4 \\
\hline Comparison feature & 0.951 & 0.853 & 2.196 & 1.183 \\
Novel features & 1.618 & 2.031 & 4.797 & 2.176 \\
No interference & 2.239 & 2.950 & 3.544 & 3.069 \\
\hline
\end{tabular}

formance below the no-interference control. Conversely, distractors incorporating the critical feature of the comparison or two novel features (the 8th and 9th harmonics) severely impaired discriminatory ability in relation to control. This effect was largely independent of the temporal position of the distractor ( $50 \mathrm{msec}$ or $8 \mathrm{sec}$ ), supporting Mercer and McKeown's (2010) notion of time-invariant interference. There were trends toward better performance at $8 \mathrm{sec}$ for the comparison-feature distractor, but no group analysis revealed this effect as being significant, and only 1 participant showed a reliable difference in performance between the two intervals for this distractor. Furthermore, distractors containing the critical feature of the comparison could even disrupt performance when they were presented $2 \mathrm{sec}$ before the standard, although novel features had a negligible influence.

The present results are extremely difficult to account for in terms of interitem confusion, since this explanation relies on the standard and distractors being perceptually similar. In the present study, the standard and distractors were fundamentally different; therefore, it seems unlikely that listeners would confuse the sequential order of these tones in the manner suggested by interitem confusion. Furthermore, since only perceptually similar tones should be confusable, the deleterious influence of novel features in Experiment 1 is incompatible with interitem confusion and is also problematic for an account based on grouping. Like interitem confusion, perceptual similarity is central to the grouping interpretation of interference, because two events that sound alike (in frequency) are liable to be merged into a single perceptual unit (Moore \& Gockel, 2002). Consequently, potent interference resulting from the novel-features distractor is unexpected from a grouping perspective. Moreover, temporally proximate events have a greater probability of being grouped into a single stream (e.g., Sussman \& Gumenyuk, 2005); thus, performance should have been worse when the distractor occurred $50 \mathrm{msec}$, rather than after $8 \mathrm{sec}$, following the offset of the standard. Discovering time-invariant interference reinforces the notion that grouping cannot adequately explain these findings. Of course, the present results do not exclude a possible role for perceptual organization in other situations, but our data strongly suggest that grouping is incapable of explaining all demonstrations of interference in auditory memory. Moreover, we dispute Jones et al.'s (1997) proposal that trace distortion and perceptual organization are two independent explanations of interference in auditory memory; instead, it seems more likely that both processes contribute to forgetting. Streaming processes may be capable of partially mediating and reducing the impact of interference (although there still appeared to be substantial forgetting in the conditions of
Jones et al., in which grouping was assumed to occur), but the memory trace can still be affected and impaired when certain distractors are presented. Thus, the present results appear to be most congruous with the feature-based interference proposed by TMM (McKeown \& Wellsted, 2009; Mercer \& McKeown, 2010).

Building on the model adjustment hypothesis (Winkler et al., 1996), the contextualized auditory memory posited by TMM serves to construct a record of the recent acoustical environment. To keep this model up to date and relevant, introducing novel sounds elicits an updating process that incorporates the latest changes within the auditory environment into short-term memory. These new sounds take precedence in a process we term updating; they are more positively weighted within the auditory record. In Experiment 1, the novel features distractor may have acted in this fashion, resulting in the significant decline in performance accuracy. However, completely novel features do not overlap with spectral features already stored within memory, and their effect is assumed to be due solely to updating, rather than to feature overwriting. But interference also can be caused when the distractor selectively overwrites features already maintained within memory, especially those crucial to the discrimination, and these new features are assumed to be weighted more positivity within the model. The discovery that distractors containing the critical feature of the comparison greatly reduced accuracy on the task in Experiment 1 is highly consistent with this notion of updating plus overwriting. And the nonsignificant impact of the noncritical-overlap and standard-feature distractors in Experiment 1 was also predicted by TMM. The model is primed to detect novel events, and so repetition of features already maintained in memory simply produces an adapted response. Turning to Experiment 2, a form of proactive feature overwriting is believed to have been responsible for the reduction in performance when distractors incorporating the comparison feature occurred $2 \mathrm{sec}$ prior to the standard. Conversely, novel features presented proactively will not overwrite the record of the subsequent standard tone (since there is no spectral overlap), and the latter will be more heavily or positively weighted through updating, thereby reducing the effect of the prior distractor. Although the plausibility of proactive overwriting has been noted in a recent study of interference in verbal short-term memory (Oberauer \& Lange, 2008), it is necessary to differentiate proactive feature overwriting from interitem confusion, which operates on the level of whole items - that is, the memory of one tone is confused with the memory of another. This explanation, therefore, posits that the actual memory itself is not distorted; only the memory for the sequential order of standard and distractors is affected. The proactive over- 
writing proffered by TMM operates on the level of specific features and indicates that the memory itself is distorted and degraded through feature interaction. Proactive interference is expected to be substantial only when there is a high degree of feature overlap between the distractor and standard tones, which is confirmed in Experiment 2.

TMM has therefore emerged as a promising account of interference in short-term auditory memory, one that links well with both the MMN literature and recent investigations into stimulus-specific adaptation. But there are some important concerns that must be discussed. For instance, the forgetting observed in the present study has been interpreted as reflecting interference within a nonverbal, shortterm store, but listeners required an average of $5.5 \mathrm{~h}$ of training before they could reliably differentiate the tones to $75 \%$ accuracy. As such, it could be suggested that, during training (and throughout testing), listeners were actually learning to create and maintain a verbal label for each of the standard stimuli. However, we are convinced that the extremely subtle spectral differences between the standard and comparison tones defied any opportunity for participants to verbally label the sounds. The participants confirmed this themselves, since none reported relying on such a strategy. Furthermore, if participants were capable of labeling the tones in this manner, the amount of interference should have been quite minimal, especially in Experiment 2, in which the distractor preceded the standard. This was not the case. Instead, the very difficulty of the discrimination demanded a prolonged period of training in order to establish reliable performance (see also studies into profile analysis, e.g., Green, 1988). Still, this raises the question of whether participants were actually developing long-term memories of the stimuli. This seems unlikely, since our approach was to train participants on the "type of change to listen for," while the actual sounds on each trial were altered around a relatively wide range. We did this by changing the fundamental frequency of the harmonics between trials (roving pitch). An analogy to this is training observers to notice very fine changes in hue while changing the actual hue of the stimulus on every trial. We are, therefore, extremely confident that long-term (verbal) memories were not involved, and comparison of participants' performance in the very first no-interference session (during testing) with the final no-interference session supports this. Ten hours of testing elapsed between these two sessions, so a substantial improvement in performance would be expected if long-term memories were involved. However, a paired samples $t$-test comparing performance across these two sessions was nonsignificant (first no-interference session performance $=.815$, last no-interference session $=.795 ; p=.634$ ). Thus, it seems highly probable that participants were forced to rely on a nonverbal and short-term representation of the standard stimulus and comparison.

That aside, certain aspects of the present results were not entirely in line with the model. TMM predicts that repetition of spectral features produces an adapted response, and distractors containing such features should, therefore, have very little influence on performance. However, although the standard feature and noncritical-overlap distractors did not significantly reduce $d^{\prime}$ in comparison with no interference, they did produce a drop in performance. This may have resulted from the intensity level of the distractors, because, although standard feature and noncritical-overlap distractors were simply a repetition of previously experienced features in terms of frequency, they were presented at a slightly greater intensity (with the 9.6-dB increment). This difference may have distorted the existing memory and produced the (nonsignificant) decline in performance. Alternatively, this finding could potentially be interpreted from a perceptual grouping perspective. Given the similar features, the standard feature and noncritical-overlap distractors may have grouped with the standard, leading to the small drop in discriminatory accuracy. Although possible, this account seems less appealing than does the adaptation explanation, since the effect of the repeated-feature distractors was almost identical at $50 \mathrm{msec}$ and $8 \mathrm{sec}$. The grouping explanation can plausibly explain the trends at $50 \mathrm{msec}$, but it becomes more difficult at the longer interval, since it would be highly unlikely that the standard and distractor would be perceptually fused after an 8-sec gap. Adaptation can explain this pattern for both intervals, but it is clear that more direct tests of this adaptation assumption are essential in future studies.

Overall then, the present findings fit very well with the predictions of TMM: Interference emerged as a timeinvariant, feature-specific phenomenon that was not determined by overall perceptual similarity. Furthermore, we believe that the "similarity-based" interference documented in many prior studies could be reinterpreted from the feature-based perspective of TMM. Still, it seems advisable to preserve some role for grouping in alleviating the influence of interference (although we are confident that grouping played no role in the present study), and there are additional findings within the literature that are not readily compatible with TMM, such as Deutsch and colleagues' experiments into lateral inhibition. Using the interpolated-tone paradigm, Deutsch (1972b) required participants to compare the pitch of standard and comparison tones across an interval containing six distractors. The critical distractor occurred in the second serial position of the interfering sequence and varied in its tonal separation from the standard (ranging from identity to whole-tone separation in 0.166-tone steps). Crucially, Deutsch found that errors on the task gradually rose as the difference between the standard and critical distractor increased up to a separation of 0.666 tone. Accuracy then began to recover, as the difference between the tones was increased further. Deutsch suggested that lateral inhibition (where the neuronal response to one tone is inhibited by the presentation of another tone) was a promising explanation of the results, and additional research by Deutsch and Feroe (1975) implied the existence of a disinhibition too. Although the overall procedure outlined by Deutsch and Feroe was similar to that reported by Deutsch (1972b), they introduced two critical distractors into the intervening tone sequence. The first of these was separated by 0.666 of a tone from the standard and was expected to produce substantial interference. The second was placed 
in the fourth position of the interpolated sequence and bore a seminal relationship to the first critical distractor. Deutsch and Feroe revealed that errors on the task were significantly reduced when the second critical distractor was separated from the first critical distractor by 0.666 tone. This appears to suggest disinhibition, whereby the second critical distractor was capable of inhibiting the first, permitting memory for the standard tone to return. Although it could be suggested that distractors 0.666 tone removed from the standard produce maximal overwriting, the discovery of disinhibition is difficult to explain within the current TMM framework. Still, perhaps Deutsch and Feroe's findings are more in line with the grouping account, since it is possible that distractors bearing a critical relationship to each other may form a single stream, distinct from the standard. Further, the "effects" reported by Deutsch and Feroe are ones within a temporal window of under $2 \mathrm{sec}$, again in line with a grouping account. Even so, some advocates of the adaptation hypothesis of MMN have also advocated a role for lateral inhibition alongside stimulus-specific adaptation (e.g., May \& Tiitinen, 2010; May et al., 1999), so perhaps it does have some part in the physiological underpinnings of auditory memory. And recently, studies of auditory evoked responses have indicated that spectral contrasts of notched noises may be produced by rather short-lived lateral inhibition of the order of $\sim 1$ sec (Okamoto, Ross, Kakigi, Kubo, \& Pantev, 2005). Psychophysical demonstrations of enhancements of spectral edges by prior sounds are provided by Carlyon (1987, 1989). For example, in Carlyon (1989), a signal within a notched-noise masker was "released" from masking when listeners had recent experience with the masker (within less than $0.5 \mathrm{sec}$ ). Carlyon argued that simple adaptation to the prior sound could not explain the finding, since it was independent of overall level of the prior stimulus, and he argued that the successive noise stimuli had been grouped perceptually, leading to a form of "popout" of the signal. Clearly, the aftereffects of prior sounds may be based on more than adaptation-based memory, but, arguably, memory-based effects may be distinguished by their enduring nature beyond a second or so (McKeown \& Wellsted, 2009).

We now wish to develop TMM as a quantitative model of updating of spectral memory. TMM is a relatively complex theory, with three major mechanisms (adaptation, updating, and overwriting), and, of central concern, is whether a quantitative version of TMM can still account for a broad range of findings, in which prior sounds influence current percepts. A further aim is to explore the similarities between verbal and nonverbal forms of memory. The present findings are well matched with certain discoveries from the verbal short-term memory literature that have also advocated feature-based interference. For instance, Oberauer (2009) presented participants with a list of four "memory" words that had to be recalled. Each of these items was followed by four distractor words, and the phonological similarity, semantic similarity, and degree of phonological overlap between memory and distractor words was varied. Analogous to the present findings, overall similarity between memory and distractor words had no adverse effect on recall, but a high degree of phonological overlap proved to be very detrimental, implicating feature overwriting. Oberauer and Lange (2008) also argued against a form of similarity-based interference. On each trial, they presented participants with four memory words, followed by four distractor consonants. Some of these consonants overlapped with phonemes in the target memory word, which significantly degraded recall. This impressive finding parallels the results of the present study, since individual features were able to overwrite specific aspects of objects currently maintained in memory. The correspondences between nonverbal and verbal forms of short-term memory noted here have also been outlined by Kaernbach (2001, 2004a, 2004b), who discovered that the lifetime, capacity, and susceptibility to interference of memory for periodic noise (which is impossible to categorize) closely resembled verbal shortterm memory. Williamson, Baddeley, and Hitch (2010) have also uncovered congruities between tonal and verbal memory in three experiments comparing serial recall of speech and music. The potent feature-based interference observed in the present study lends further support to this notion and suggests that additional exploration of the relationship between verbal and nonverbal forms of auditory memory is warranted.

\section{AUTHOR NOTE}

We thank Jian Bi for providing the S-PLUS code used to calculate $d^{\prime}$ and its associated variance. We also thank three anonymous reviewers for their valuable comments on an earlier version of the manuscript. Correspondence concerning this article should be addressed to T. Mercer, Institute of Psychological Sciences, University of Leeds, Leeds LS2 9JT, England (e-mail: t.mercer04@leeds.ac.uk).

\section{REFERENCES}

Alvarez, G. A., \& Cavanagh, P. (2008). Visual short-term memory operates more efficiently on boundary features than on surface features. Perception \& Psychophysics, 70, 346-364. doi:10.3758/PP.70.2.346

BACHEM, A. (1954). Time factors in relative and absolute pitch determination. Journal of the Acoustical Society of America, 26, 751-753. doi:10.1121/1.1907411

Berman, M. G., Jonides, J., \& Lewis, R. L. (2009). In search of decay in verbal short-term memory. Journal of Experimental Psychology: Learning, Memory, \& Cognition, 35, 317-333. doi:10.1037/ a0014873

Berti, S., Münzer, S., Schröger, E., \& Pechmann, T. (2006). Different interference effects in musicians and a control group. Experimental Psychology, 53, 111-116. doi:10.1027/1618-3169.53.2.111

BI, J. (2002). Variance of $d^{\prime}$ for the same-different method. Behavior Research Methods, Instruments, \& Computers, 34, 37-45.

Brosch, M., Schulz, A., \& Scheich, H. (1999). Processing of sound sequences in macaque auditory cortex: Response enhancement. Journal of Neurophysiology, 82, 1542-1559.

Camos, V., Lagner, P., \& Barrouillet, P. (2009). Two maintenance mechanisms of verbal information in working memory. Journal of Memory \& Language, 61, 457-469. doi:10.1016/j.jml.2009.06.002

CARlyon, R. P. (1987). A release from masking by continuous, random, notched noise. Journal of the Acoustical Society of America, 81, 418426. doi:10.1121/1.395117

Carlyon, R. P. (1989). Changes in the masked thresholds of brief tones produced by prior bursts of noise. Hearing Research, 41, 223-235. doi:10.1016/0378-5955(89)90014-2

Clément, S., Demany, L., \& Semal, C. (1999). Memory for pitch versus memory for loudness. Journal of the Acoustical Society of America, 106, 2805-2811. doi:10.1121/1.428106 
Cowan, N., \& AuBuchon, A. M. (2008). Short-term memory loss over time without retroactive stimulus interference. Psychonomic Bulletin \& Review, 15, 230-235. doi:10.3758/PBR.15.1.230

Cowan, N., Saults, J. S., \& Nugent, L. D. (1997). The role of absolute and relative amounts of time in forgetting within immediate memory: The case of tone-pitch comparisons. Psychonomic Bulletin \& Review, 4, 393-397.

Cowan, N., Saults, J. S., \& Nugent, L. D. (2001). The ravages of absolute and relative amounts of time on memory. In H. L. Roediger III, J. S. Nairne, I. Neath, \& A. M. Surprenant (Eds.), The nature of remembering: Essays in honor of Robert G. Crowder (pp. 315330). Washington, DC: American Psychological Association. doi:10 1037/10394-017

DAI, H., SchARF, B., \& BuUs, S. (1991). Effective attenuation of signals in noise under focused attention. Journal of the Acoustical Society of America, 89, 2837-2842. doi:10.1121/1.400721

Demany, L., \& Semal, C. (2008). The role of memory in auditory perception. In W. A. Yost, A. N. Popper, \& R. F. Fays (Eds.), Auditory perception of sound sources (pp. 77-113). New York: Springer. doi:10.1007/978-0-387-71305-2_4

Deutsch, D. (1970). Tones and numbers: Specificity of interference in immediate memory. Science, 168, 1604-1605. doi:10.1126/ science.168.3939.1604

DEuTsCH, D. (1972a). Effect of repetition of standard and comparison tones on recognition memory for pitch. Journal of Experimental Psychology, 93, 156-162. doi:10.1037/h0032496

Deutsch, D. (1972b). Mapping of interactions in the pitch memory store. Science, 175, 1020-1022. doi:10.1126/science.175.4025.1020

Deutsch, D. (1978a). Delayed pitch comparisons and the principle of proximity. Perception \& Psychophysics, 23, 227-230.

Deutsch, D. (1978b). Interactive effects in memory for harmonic intervals. Perception \& Psychophysics, 24, 7-10.

Deutsch, D. (1982). The influence of melodic context on pitch recognition judgment. Perception \& Psychophysics, 31, 407-410.

Deutsch, D. (1984). Memory for nonverbal auditory information: A link between behavioral and physiological studies. In L. R. Squire \& N. Butters (Eds.), Neuropsychology of memory (pp. 45-54). New York: Guilford.

Deutsch, D. (1999). The processing of pitch combinations. In D. Deutsch (Ed.), The psychology of music (2nd ed., pp. 349-411). San Diego: Academic Press.

Deutsch, D., \& Feroe, J. (1975). Disinhibition in pitch memory. Perception \& Psychophysics, 17, 320-324.

Deutsch, D., \& Roll, P. L. (1974). Error patterns in delayed pitch comparison as a function of relational context. Journal of Experimental Psychology, 103, 1027-1034. doi:10.1037/h0037359

DuRLACH, N. I., \& BRAIDA, L. D. (1969). Intensity perception: I. Preliminary theory of intensity resolution. Journal of the Acoustical Society of America, 46, 372-383. doi:10.1121/1.1911699

Elliott, L. L. (1970). Pitch memory for short tones. Perception \& Psychophysics, 8, 379-384.

Fougnie, D., \& Marois, R. (2009). Dual-task interference in visual working memory: A limitation in storage capacity but not in encoding or retrieval. Attention, Perception, \& Psychophysics, 71, 1831-1841. doi:10.3758/APP.71.8.1831

Garrido, M. I., Kilner, J. M., Stephan, K. E., \& Friston, K. J. (2009). The mismatch negativity: A review of underlying mechanisms. Clinical Neurophysiology, 120, 453-463. doi:10.1016/j.clinph .2008 .11 .029

Gourevitch, V., \& Galanter, E. (1967). A significance test for one-parameter isosensitivity functions. Psychometrika, 32, 25-33. doi:10.1007/BF02289402

Green, D. M. (1988). Profile analysis: Auditory intensity discrimination. New York: Oxford University Press.

Green, D. M., Kidd, G., JR., \& PiCARdi, M. C. (1983). Successive versus simultaneous comparison in auditory intensity discrimination. Journal of the Acoustical Society of America, 73, 639-643. doi:10.1121/1.389009

Green, T. J., \& McKeown, J. D. (2001). Capture of attention in selective frequency listening. Journal of Experimental Psychology: Human Perception \& Performance, 27, 1197-1210. doi:10.1037/0096 $-1523.27 .5 .1197$

Green, T. [J.], \& McKeown, [J.] D. (2007). The role of auditory mem- ory traces in attention to frequency. Perception \& Psychophysics, 69 , 942-951.

Hafter, E. R., Schlauch, R. S., \& TANG, J. (1993). Attending to auditory filters that were not stimulated directly. Journal of the Acoustical Society of America, 94, 743-747. doi:10.1121/1.408203

HARRIS, J. D. (1952). The decline of pitch discrimination with time. Journal of Experimental Psychology, 43, 96-99. doi:10.1037/h0057373

Horváth, J., Czigler, I., Jacobsen, T., Maess, B., Schröger, E., \& Winkler, I. (2008). MMN or no MMN: No magnitude of deviance effect on the MMN amplitude. Psychophysiology, 45, 60-69. doi:10.1111/j.1469-8986.2007.00599.x

Hourihan, K. L., Ozubko, J. D., \& MacLeod, C. M. (2009). Directed forgetting of visual symbols: Evidence for nonverbal selective rehearsal. Memory \& Cognition, 37, 1059-1068. doi:10.3758/ MC.37.8.1059

Hübner, R., \& HAFTER, E. R. (1995). Cuing mechanisms in auditory signal detection. Perception \& Psychophysics, 57, 197-202.

JäÄskeläınen, I. P., Ahveninen, J., Bonmassar, G., Dale, A. M., ILmoniemi, R. J., LeVÄNEN, S., ET AL. (2004). Human posterior auditory cortex gates novel sounds to consciousness. Proceedings of the National Academy of Sciences, 101, 6809-6814. doi:10.1073/ pnas.0303760101

Jones, D. M., Macken, W. J., \& Harries, C. (1997). Disruption of short-term recognition memory for tones: Streaming or interference? Quarterly Journal of Experimental Psychology, 50A, 337-357. doi:10.1080/713755707

Jonides, J., Lewis, R. L., Nee, D. E., Lustig, C. A., Berman, M. G., \& Moore, K. S. (2008). The mind and brain of short-term memory. Annual Review of Psychology, 59, 193-224. doi:10.1146/annurev .psych.59.103006.093615

KaERnBach, C. (2001). Parameters of echoic memory. In E. Sommerfeld, R. Kompass, \& T. Lachmann (Eds.), Proceedings of the seventeenth annual meeting of the International Society for Psychophysics (pp. 105-110). Lengerich: Pabst Science.

KAERNBACH, C. (2004a). Auditory sensory memory and short-term memory. In C. Kaernbach, E. Schröger, \& H. Müller (Eds.), Psychophysics beyond sensation: Laws and invariants of human cognition (pp. 331-348). Mahwah, NJ: Erlbaum.

KAERNBACH, C. (2004b). The memory of noise. Experimental Psychology, 51, 240-248. doi:10.1027/1618-3169.51.4.240

KaERnBACH, C., \& Schlemmer, K. (2008). The decay of pitch memory during rehearsal. Journal of the Acoustical Society of America, 123, 1846-1849. doi:10.1121/1.2875365

KidD, G., JR., \& Mason, C. R. (1992). A new technique for measuring spectral shape discrimination. Journal of the Acoustical Society of America, 91, 2855-2864. doi:10.1121/1.402966

Lewandowsky, S., Brown, G. D. A., Wright, T., \& Nimmo, L. M. (2006). Timeless memory: Evidence against temporal distinctiveness models of short-term memory for serial order. Journal of Memory \& Language, 54, 20-38. doi:10.1016/j.jml.2005.08.004

Lewandowsky, S., Oberauer, K., \& Brown, G. D. A. (2009). No temporal decay in verbal short-term memory. Trends in Cognitive Sciences, 13, 120-126. doi:10.1016/j.tics.2008.12.003

Lu, Z.-L., Williamson, S. J., \& Kaufman, L. (1992). Behavioral lifetime of human auditory sensory memory predicted by physiological measures. Science, 258, 1668-1670. doi:10.1126/science.1455246

Macmillan, N. A., \& Creelman, C. D. (2005). Detection theory: A user's guide (2nd ed.). Mahwah, $\mathrm{NJ}$ : Erlbaum.

Marascuilo, L. A. (1970). Extensions of the significance test for oneparameter signal detection hypotheses. Psychometrika, 35, 237-243. doi:10.1007/BF02291265

Massaro, D. W. (1970). Consolidation and interference in the perceptual memory system. Perception \& Psychophysics, 7, 153-156.

Massaro, D. W. (1972). Preperceptual images, processing time and perceptual units in auditory perception. Psychological Review, 79, 124-145. doi: $10.1037 / \mathrm{h} 0032264$

May, P. J. C., \& Tirtinen, H. (2007). The role of adaptation-based memory in auditory cortex. International Congress Series, 1300, 53-56. doi:10.1016/j.ics.2007.01.051

May, P. J. C., \& Tittinen, H. (2010). Mismatch negativity (MMN), the deviance-elicited auditory deflection, explained. Psychophysiology, 47, 66-122. doi:10.1111/j.1469-8986.2009.00856.x

May, P. J. C., Timtinen, H., Ilmoniemi, R. J., Nyman, G., Taylor, 
J. G., \& NÄÄTÄNEN, R. (1999). Frequency change detection in human auditory cortex. Journal of Computational Neuroscience, 6, 99-120. doi:10.1023/A:1008896417606

McGaUgh, J. L. (2000). Memory-A century of consolidation. Science, 287, 248-251. doi:10.1126/science.287.5451.248

McKeown, D., \& Wellsted, D. (2009). Auditory memory for timbre. Journal of Experimental Psychology: Human Perception \& Performance, 35, 855-875. doi:10.1037/a0013708

Mercer, T., \& McKeown, D. (2010). Interference in short-term auditory memory. Quarterly Journal of Experimental Psychology, 63, 1256-1265. doi:10.1080/17470211003802467

Michael, G. A. (2007). A significance test of interaction in 2xK designs with proportions. Tutorials in Quantitative Methods for Psychology, 3, $1-7$

Moore, B. C. J., \& Gockel, H. (2002). Factors influencing sequential stream segregation. Acta Acustica United With Acustica, 88, 320-333.

NÄÄTÄNEN, R., JACOBSEN, T., \& WiNKLER, I. (2005). Memory-based or afferent processes in mismatch negativity (MMN): A review of the evidence. Psychophysiology, 42, 25-32. doi:10.1111/j.1469 $-8986.2005 .00256 . \mathrm{x}$

NAIRNE, J. S. (2003). Sensory and working memory. In I. B. Weiner (Series Ed.) \& A. F. Healy \& R. W. Proctor (Vol. Eds.), Handbook of psychology: Vol. 4. Experimental psychology (pp. 423-444). New York: Wiley.

NosOFSKy, R. M. (1983). Shifts of attention in the identification and discrimination of intensity. Perception \& Psychophysics, 33, 103-112.

OBERAUER, K. (2009). Interference between storage and processing in working memory: Feature overwriting, not similarity-based competition. Memory \& Cognition, 37, 346-357. doi:10.3758/MC.37.3.346

OBERAUER, K., \& LANGE, E. B. (2008). Interference in verbal working memory: Distinguishing similarity-based competition, feature overwriting, and feature migration. Journal of Memory \& Language, $\mathbf{5 8}$, 730-745. doi:10.1016/j.jml.2007.09.006

Oberle, C. D., \& AmaZEEn, E. L. (2003). Independence and separability of volume and mass in the size-weight illusion. Perception \& Psychophysics, 65, 831-843.

Oкамото, H., Ross, B., Kakigi, R., Kubo, T., \& Pantev, C. (2005). The time course of $\mathrm{N} 1 \mathrm{~m}$ decline caused by exposure to noise with strong spectral contrasts. International Congress Series, 1278, 23-26. doi:10.1016/j.ics.2004.11.006

Pechmann, T., \& Mohr, G. (1992). Interference in working memory for tonal pitch: Implications for a working-memory model. Memory \& Cognition, 20, 314-320.

Ries, D. T., \& DiGiovanni, J. J. (2007). Release from interference in auditory working memory for pitch. Hearing Research, 230, 64-72. doi:10.1016/j.heares.2007.04.003

Ries, D. T., \& DiGiovanNi, J. J. (2009). Effects of recurrent tonal information on auditory working memory for pitch. Hearing Research, 255, 14-21. doi:10.1016/j.heares.2009.05.002

RUUSUVIRTA, T. (2000). Proactive interference of a sequence of tones in a two-tone pitch comparison task. Psychonomic Bulletin \& Review, 7, 327-331.

RuUsuvirta, T., Astikainen, P., \& Wikgren, J. (2002). Proactive interference of differently ordered tone sequences with the accuracy and speed of two-tone frequency comparisons. Music Perception, 19, 551-563. doi:10.1525/mp.2002.19.4.551

Ruusuvirta, T., Wikgren, J., \& Astikainen, P. (2008). Proactive interference in a two-tone pitch-comparison task without additional interfering tones. Psychological Research, 72, 74-78. doi:10.1007/ s00426-006-0094-y

Semal, C., Demany, L., Ueda, K., \& Hallé, P.-A. (1996). Speech versus nonspeech in pitch memory. Journal of the Acoustical Society of America, 100, 1132-1140. doi:10.1121/1.416298
Snyder, J. S., Carter, O. L., Lee, S.-K., Hannon, E. E., \& Alain, C. (2008). Effects of context on auditory stream segregation. Journal of Experimental Psychology: Human Perception \& Performance, 34, 1007-1016. doi:10.1037/0096-1523.34.4.1007

StARR, G. E., \& PitT, M. A. (1997). Interference effects in short-term memory for timbre. Journal of the Acoustical Society of America, 102, 486-494. doi:10.1121/1.419722

Sussman, E. S., \& GumenyuK, V. (2005). Organization of sequential sounds in auditory memory. NeuroReport, 16, 1519-1523. doi:10.1097/01.wnr.0000177002.35193.4c

Sussman, E. [S.], \& Winkler, I. (2001). Dynamic sensory updating in the auditory system. Cognitive Brain Research, 12, 431-439. doi:10.1016/S0926-6410(01)00067-2

TarkKa, I. M., Lehtovirta, M., Soininen, H., Pä̈̈KKönen, A., Karhu, J., \& Partanen, J. (2002). Auditory adaptation is differentially impaired in familial and sporadic Alzheimer's disease. Biomedicine \& Pharmacotherapy, 56, 45-49. doi:10.1016/S0753 $-3322(01) 00149-4$

UEDA, K. (2004). Short-term auditory memory interference: The Deutsch demonstration revisited. Acoustical Science \& Technology, 25, 457-467. doi:10.1250/ast.25.457

Ulanovsky, N., Las, L., Farkas, D., \& Nelken, I. (2004). Multiple time scales of adaptation in auditory cortex neurons. Journal of Neuroscience, 24, 10440-10453. doi:10.1523/JNEUROSCI.1905-04.2004

Ulanovsky, N., Las, L., \& Nelken, I. (2003). Processing of lowprobability sounds by cortical neurons. Nature Neuroscience, $\mathbf{6}, 391-$ 398. doi: $10.1038 / \mathrm{nn} 1032$

Visscher, K. M., Kahana, M., \& Sekuler, R. (2009). Trial-to-trial carryover in auditory short-term memory. Journal of Experimental Psychology: Learning, Memory, \& Cognition, 35, 46-56. doi:10.1037/ a0013412

von DER Behrens, W., BäUerle, P., Kössl, M., \& Gaese, B. H. (2009). Correlating stimulus-specific adaptation of cortical neurons and local field potentials in the awake rat. Journal of Neuroscience, 29, 1383713849. doi:10.1523/JNEUROSCI.3475-09.2009

Williamson, V. J., Baddeley, A. D., \& Нitch, G. J. (2010). Musicians' and nonmusicians' short-term memory for verbal and musical sequences: Comparing phonological similarity and pitch proximity. Memory \& Cognition, 38, 163-175. doi:10.3758/MC.38.2.163

WINKLER, I. (2007). Interpreting the mismatch negativity. Journal of Psychophysiology, 21, 147-163. doi:10.1027/0269-8803.21.34.147

WINKLER, I., \& CoWAN, N. (2005). From sensory to long-term memory: Evidence from auditory memory reactivation studies. Experimental Psychology, 52, 3-20. doi:10.1027/1618-3169.52.1.3

WinKLER, I., KARMOS, G., \& NäÄTÄNEN, R. (1996). Adaptive modeling of the unattended acoustic environment reflected in the mismatch negativity event-related potential. Brain Research, 742, 239-252. doi:10.1016/S0006-8993(96)01008-6

Winkler, I., Sussman, E., Tervaniemi, M., Horváth, J., Ritter, W., \& NäÄTÄNen, R. (2003). Preattentive auditory context effects. Cognitive, Affective, \& Behavioral Neuroscience, 3, 57-77. doi:10.3758/ CABN.3.1.57

WIXTED, J. T. (2004). The psychology and neuroscience of forgetting. Annual Review of Psychology, 55, 235-269. doi:10.1146/annurev .psych.55.090902.141555

ZHANG, W., \& LUCK, S. J. (2009). Sudden death and gradual decay in visual working memory. Psychological Science, 20, 423-428. doi:10.1111/j.1467-9280.2009.02322.x

(Manuscript received March 31, 2010; revision accepted for publication June 15, 2010.) 